\title{
LA RESPONSABILIDAD SOCIAL EMPRESARIAL (RSE) Y SU INFLUENCIA EN LOS RESULTADOS ECONÓMICOS DE LAS EMPRESAS INDUSTRIALES DE LA CIUDAD DETACNA, PERIODO: 2010 - 2011
}

\author{
CORPORATE SOCIAL RESPONSIBILITY (CSR) AND ITS INFLUENCE ON \\ ECONOMIC PERFORMANCE OF INDUSTRIAL ENTERPRISES TACNA \\ CITY, PERIOD: 2010-2011
}

\author{
Luis Roccheti Herrera'; Elizabeth Medina Soto²; Jesús Olivera Cáceres ${ }^{3}$; \\ Gonzalo Gambini Quezada ${ }^{4}$
}

\begin{abstract}
RESUMEN
El presente artículo tiene por finalidad evaluar si la Responsabilidad Social Empresarial (RSE) que influye en los resultados económicos de las empresas industriales de la ciudad de Tacna, periodo:2010-2011.

Actualmente en la región Tacna existen empresas industriales en regular medida, por lo que resulta relevante la necesidad de contar con una estrategia de RSE que confirme el compromiso que las empresas tienen, el de contribuir y colaborar con su entorno. En la investigación se elaboró un cuestionario a fin de verificar la influencia de la responsabilidad en los resultados económicos de la empresa. Luego se analizó si las empresas industriales aplican prácticas o políticas RSE. Enfatizamos en la reputación cuantía de inversión en la RSE, modalidad y medición de sus programas y procesos. Finalmente, en cómo la empresa mide el impacto de sus operaciones a nivel económico, social y ambiental. Con este propósito se utilizó el método descriptivo, explicativo, cuantitativo.
\end{abstract}

Palabras clave: Responsabilidad social empresarial, Reputación social, Empresas industriales, Inversión en RSE.

\section{ABSTRACT}

This scientific paper is designed to assess whether Corporate Social Responsibility (CSR) influences economic performance of industrial enterprises of the city of Tacna, period:2010-2011.

Currently, the region has Tacna regulate industrial enterprises measure, so it is relevant the need for a CSR strategy, to confirm the commitment of the company to contribute and collaborate with their environment. In the research a questionnaire was developed to verify the influence of the responsibility for the economic performance of the company. We analyzed whether the industrial practices or policies apply Corporate Social Responsibility (CSR), how to consider CSR, which high lighted the social reputation, and how much they invest in CSR and If They measure and evaluate their programs and processes Corporate Social Responsibility. Also, if the company measures the impact of its operations on economic, social and environmental. We used a descriptive method, explanatory, quantitative.

Keywords: corporate social responsibility, reputation, social, industrial, investment in CSR.

\section{INTRODUCCIÓN}

Hoy en día, la Responsabilidad Social Empresarial (en adelante RSE) en nuestro país se canaliza mediante un conjunto de iniciativas, las cuales buscan enfrentar este valor de conciencia empresarial. El objetivo no es sólo la legitimación social, sino también tratar de alcanzar la tan ansiada reputación social corporativa en la sociedad.

Contar con una estrategia de RSE, confirma el compromiso que tiene la empresa de contribuir y colaborar con su entorno.

La responsabilidad social corporativa (RSC) y la Reputación corporativa (RC) son dos conceptos diferentes, pero que se encuentran ligados.

La RSC es la gestión del relacionamiento con grupos de interés, entre ellos colaboradores, proveedores, clientes, comunidad y entorno social, lo cual significa entender sus intereses $y$ preocupaciones, administrar los impactos

\footnotetext{
'Ingeniero Comercial, Magíster en Dirección de Empresas. Facultad de Ciencias Jurídicas y Empresariales de la UNJBG.

${ }^{2}$ Contadora Pública, Magíster en Contabilidad y Auditoría. Facultad de Ciencias Jurídicas y Empresariales de la UNJBG.

${ }^{3}$ Ingeniero Economista, Magíster en Docencia Universitaria, Doctor en Administración. Facultad de Ciencias Jurídicas y Empresariales de la UNJBG.

${ }^{4}$ Economista, Magister en Gestión Empresarial. Facultad de Ciencias Jurídicas y Empresariales de la UNJBG.
} 
negativos y positivos e ir más allá de ser un buen vecino, mientras que la $\mathrm{RC}$ viene, en parte, como producto del desempeño de la RSC, conjuntamente con la identidad de la marca. Es esencialmente pública y corresponde a lo que la gente está diciendo de nosotros.

La RC es el activo más importante de la empresa, pues crea y sostiene el valor de la marca, el precio de la acción, la confianza de los clientes y proveedores, la credibilidad ante las autoridades y la retención de los mejores empleados.

El presente trabajo de investigación, relacionado a la Responsabilidad Social Empresarial (RSE) en las empresas industriales de la ciudad de Tacna, busca determinar si la responsabilidad social es parte de la estrategia organizacional de las empresas. Con ese fin, se indagó, cuáles acciones se desarrollan y si hay coherencia entre las acciones y los valores de las empresas industriales. Las empresas interpretan la Responsabilidad Social Empresarial como la responsabilidad que asumen frente a todos los grupos de interés, pero en la práctica atienden expectativas de los de ellos.

También se estableció si existe coherencia entre los valores y las acciones realizadas por las empresas industriales, luego si es verdad que existe desconocimiento frente al tema, dado que aunque interpretan que la RSE contribuye a la competitividad de las empresas y hacen importantes esfuerzos en este sentido, no la incluyen como parte de la estrategia.

Merece resaltar que se debe considerar la aplicación de la RSE como una inversión, que puede ser recuperada en el largo plazo, lo cual permitiría a la empresa recibir una recompensa, en términos de credibilidad y confianza, y la sociedad obtendría un doble beneficio, porque a esta también le interesa que las empresas sean exitosas.

La muestra de estudio de la presente investigación está constituida por las empresas industriales de la ciudad de Tacna, por lo que las unidades de observación son los Gerentes y/o entes de las referidas empresas; las variables de estudio fueron la responsabilidad social empresarial y los resultados económicos. El estudio se realizó en la ciudad de Tacna.

Se debe partir reconociendo que la responsabilidad significa asumir las consecuencias de los actos realizados, lo que traducido a nivel empresarial significa responder ante la sociedad por los resultados que se generan a partir de las decisiones adoptadas y las acciones que se desarrollan por efecto de ellas.

Las empresas no son ni directa ni exclusivamente responsables de resolver los problemas sociales; pero si lo son, en principio, de solucionar los problemas que con su operación le ocasionan a la sociedad. Es claro que el principal rol que debe jugar el sector privado como miembro de la sociedad es el de generar utilidades; también que la contraprestación económica y redistributiva que deben cumplir las empresas es la de pagar los impuestos que les corresponde, para que el gobierno, con criterio de equidad, los invierta en la generación de bienestar social, garantizando la combinación de la calidad y el nivel de vida de los ciudadanos.

Las organizaciones intervienen en el entorno y por eso son responsables de su influencia. Las empresas son parte de la sociedad, y como miembros de ésta, tienen derechos que exigir y deberes que cumplir.

Las empresas, así como las demás organizaciones sociales, no pueden responsabilizar de manera exclusiva al Estado, acerca del funcionamiento social, de la equidad en la distribución del ingreso y del bienestar. En cambio, tienen que compartir con el Estado la construcción de la sociedad civil.

Como miembros de la sociedad, las compañías, a través de sus directivos, tienen el derecho de buscar la generación de valor con el propósito de obtener el nivel de rentabilidad que esperan, pero, a su vez, tienen el deber de lograrlo sin ocasionar daño o afectación negativa a los miembros de la sociedad en la que están inmersas y, además, deben garantizar la distribución equitativa del valor producido. Como bien lo plantea Idelfonso Camacho(2005), las empresas deberían ser tan efectivas para generar valor, como para distribuirlo de manera equitativa entre quienes contribuyeron a su generación.

\subsection{Antecedentes de la RSE}

Los primeros aportes de la RSE aparece en el libro Border Social Responsibilities of the Business man (1953), sin embargo existen otras referencias anteriores.

Desde los años cincuenta se han venido realizando investigaciones sobre responsabilidad social corporativa, que buscan responder a las expectativas de la comunidad, para ayudar a crear una mejor sociedad.

Guidi (2006) menciona que el debate sobre los compromisos sociales y filantrópicos que deberían asumir las empresas se remonta a los años setenta. 
El origen de la discusión sobre RSE está ligado al llamado de atención que significó el Informe Bruntland (1987), al definir el desarrollo sustentable como "el desarrollo que satisface las necesidades actuales sin comprometer la capacidad de las generaciones futuras para atender a sus necesidades".

El panorama teórico sobre el cual se apoya la RSE se basa en La teoría de la agencia (Friedman, 1970), The Stakeholders Theory (Donaldson y Preston, 1995), Perspectiva de la ciudadanía corporativa (Waddock, 2005; Wood y Lodgson, 2002), Desempeño social corporativo (Wood, 1991), La teoría basada en los recursos (Barney, 1991); Perspectiva estratégica (Achatan, 2004). La teoría de convenciones con énfasis en la "cooperación cívica" (Ponte y Gibbon, 2003) y, finalmente. La perspectiva del Commongood (Argandoña, 1998; Melé, 2002).

\subsection{Definición de la RSE}

El término Responsabilidad Social Empresarial se empieza a utilizar durante el Foro Económico Mundial de 1999, por el secretario general de la Organización de las Naciones Unidas (ONU). Sin embargo, a pesar que muchas organizaciones lo han empleado, no existe un consenso en los distintos organismos internacionales, sobre una definición precisa.

Algunas instituciones han desarrollado definiciones operativas, como la OCDE (2000), que emplea el concepto de responsabilidad corporativa y lo define como "Las acciones desarrolladas por negocios para consolidar sus relaciones con las sociedades en las que actúan".

La Comisión Europea en el Libro Verde: "Fomentar un marco europeo para la responsabilidad de las empresas", publicado por la Comisión Europea en el 2001, defina la RSE como "la integración voluntaria, por parte de las empresas, de las preocupaciones sociales y ambientales en sus operaciones empresariales y en sus relaciones con sus interlocutores (Stakeholders)".

El Instituto Ethos de Empresas y Responsabilidad Social (2007) de Brasil define a la Responsabilidad Social Empresarial, como "La forma de gestión que se define por la relación ética y transparente de la empresa con todos los públicos con los cuales se relaciona, y por el establecimiento de metas empresariales, compatibles con el desarrollo sustentable de la sociedad, al preservar recursos ambientales y culturales para las generaciones futuras, y al respetar la diversidad y promover la reducción de las desigualdades sociales".

La Red Forum Empresa (2011) señala que, no existe una definición única de la RSE. Se trata de una visión de los negocios que incorpora el respeto por los valores éticos, las personas, las comunidades y el medio ambiente.

Por lo expuesto, podemos decir que no existe una definición generalizada respecto a la RSE. En noviembre del 2010, con la participación de más de 100 países la ISO 2600 , se establece la primera herramienta que integra una guía de cómo aplicar la RSE en toda clase de organizaciones. Considera que debe verse como una nueva forma de operar las actividades de la organización basada en procesos, estructuras y recursos existentes, y bajo la cual convergen accionistas, proveedores, comunidad, medio ambiente, gobierno, clientes y colaboradores (Remy, 2011). La norma define a la RSE como "la responsabilidad de una organización por los impactos que sus decisiones y actividades ocasionan en la sociedad y el medio ambiente, a través de un comportamiento transparente y ético que contribuya al desarrollo sostenible, la salud y el bienestar de la sociedad, tome en consideración las expectativas de sus partes interesadas, cumpla con la legislación aplicable y sea coherente con la normativa internacional de comportamiento y esté integrada en toda la organización y se lleve a la práctica en sus relaciones" (Duran, 2008; Puterman, 2010).

De estas definiciones se puede decir que la RSE implica que las organizaciones deben cumplir de forma estricta las normas legales vigentes, y asumir voluntariamente una serie de comportamientos éticos (preocupaciones sociales, laborales, medioambientales, etc.), derivados de las relaciones que mantienen con sus grupos de interés o stakeholders y que suponen un "plus" de responsabilidad (De Castro, 2005).

\section{MATERIALES Y MÉTODOS}

\subsection{Material}

En el presente trabajo, la unidad de análisis fueron las empresas industriales de la ciudad de Tacna, de las que se tomó una muestra de 12 empresas.

\subsection{Metodología}

Este trabajo es de tipo cuantitativo, dïseño no experimental y descriptivo. Se utilizó la encuesta y el cuestionario. Para realizar el procesamiento, análisis y presentación de los datos se empleó el paquete estadístico SPSS 20. 


\section{RESULTADOS}

\subsection{La organización tiene prácticas/políticas de RSE}

Como se observa en la Tabla $\mathrm{N}^{\circ} \mathrm{O} 1$, el $58,3 \%$ de los gerentes $\mathrm{y} / \mathrm{o}$ del personal administrativo de las empresas industriales de la ciudad de Tacna manifiestan que su organización sí tiene prácticas/políticas de RSE.

En contraste, un $41,7 \%$ de los gerentes y/o del personal administrativo, dicen que su organización no tiene prácticas/políticas de RSE.

Tabla $\mathrm{N}^{\circ}$ 01:Porcentaje de prácticas/ políticas de RSE en la organización.

\begin{tabular}{|c|r|r|r|}
\hline Categoría & Frecuencia & Porcentaje & $\begin{array}{l}\text { Porcentaje } \\
\text { acumulado }\end{array}$ \\
\hline Sí & 7 & 58,3 & 58,3 \\
\hline No & 5 & 41,7 & 100,0 \\
\hline Total & 12 & 100,0 & \\
\hline
\end{tabular}

Fuente: Matriz de sistematización de datos.

\subsection{La RSE es un asunto de relaciones públicas muy importante}

Como se observa en la Tabla $\mathrm{N}^{\circ} \mathrm{O} 2$, el $58,3 \%$ de los gerentes y/o del personal administrativo de las empresas industriales de la ciudad de Tacna manifiestan que están poco de acuerdo con que la RSE sea un asunto de relaciones públicas muy importantes. En contraste, el $33,3 \%$ de los gerentes $\mathrm{y} / \mathrm{o}$ el personal administrativo, dicen estar muy de acuerdo que la RSE es un asunto de relaciones públicas muy importante. Sólo el 8,3\% de los gerentes y/o personal administrativo de las empresas industriales manifiestan estar de acuerdo.

Tabla $\mathrm{N}^{\circ}$ o2: Porcentaje de importancia de las RSE en las relaciones públicas.

\begin{tabular}{|c|r|r|r|}
\hline Categoría & Frecuencia & Porcentaje & $\begin{array}{r}\text { Porcentaje } \\
\text { Acumulado }\end{array}$ \\
\hline Poco de acuerdo & 7 & 58,3 & 58,3 \\
\hline De acuerdo & 1 & 8,3 & 66,7 \\
\hline Muy de acuerdo & 4 & 33,3 & 100,0 \\
\hline Total & 12 & 100,0 & \\
\hline
\end{tabular}

Fuente: Matriz de sistematización de datos

\subsection{La responsabilidad social es vital para la sustentabilidad y rentabilidad de cualquier empresa}

Como se observa en la Tabla $\mathrm{N}^{\circ} 03$, el 16,7\% de los gerentes y/o del personal administrativo de las empresas industriales de la ciudad de Tacna manifiestan estar poco de acuerdo que la RSE es vital para la sustentabilidad y rentabilidad de cualquier empresa. En contraste, el $58,3 \%$ de gerentes $\mathrm{y} / \mathrm{o}$ del personal administrativo, dicen estar de acuerdo con la RSE es vital para la sustentabilidad y rentabilidad de cualquier empresa. Sólo el $25 \%$ de los gerentes y/o del personal administrativo manifiestan estar muy de acuerdo respecto al tema.

Tabla $\mathrm{N}^{\circ}$ 03: Porcentaje de importancia vital para la sustentabilidad y rentabilidad de cualquier empresa con la RSE.

\begin{tabular}{|c|r|r|r|}
\hline \multicolumn{1}{|c|}{ Categoría } & Frecuencia & Porcentaje & $\begin{array}{r}\text { Porcentaje } \\
\text { Acumulado }\end{array}$ \\
\hline Poco de acuerdo & 2 & 16,7 & 16,7 \\
\hline De acuerdo & 7 & 58,3 & 75,0 \\
\hline Muy de acuerdo & 3 & 25,0 & 100,0 \\
\hline Total & 12 & 100,0 & \\
\hline
\end{tabular}

Fuente: Matriz de sistematización de datos.

\subsection{En el clima económico actual las actividades de RSE tienen una prioridad baja}

Como se observa en la Tabla $\mathrm{N}^{\circ} 04$, el $25 \%$ de los gerentes $\mathrm{y} / \mathrm{o}$ del personal administrativo de las empresas industriales de la ciudad de Tacna consideran que están en desacuerdo. En el clima económico actual las actividades de RSE tienen una prioridad baja.

En contraste, el $50 \%$ de los gerentes y/o del personal administrativo consideran que están de acuerdo que en el clima económico actual, las actividades de RSE tienen una prioridad baja, mientras que sólo el $25 \%$ de los gerentes y/o del personal administrativo, dice estar poco de acuerdo.

Tabla $\mathbf{N}^{\circ}$ 04: Porcentaje de aceptación de la prioridad de las actividades de RSE en el clima económico actual.

\begin{tabular}{|c|r|r|r|}
\hline Categoría & Frecuencia & Porcentaje & $\begin{array}{c}\text { Porcentaje } \\
\text { acumulado }\end{array}$ \\
\hline En desacuerdo & 3 & 25,0 & 25,0 \\
\hline Poco de acuerdo & 3 & 25,0 & 50,0 \\
\hline De acuerdo & 6 & 50,0 & 100,0 \\
\hline Total & 12 & 100,0 & \\
\hline
\end{tabular}

Fuente: Matriz de sistematización de datos

\subsection{Consideración de la responsabilidad social como un asunto de "reputación social"}

Como se observa en la Tabla $\mathrm{N}^{\circ} \mathrm{O} 5$, el $25 \%$ de los gerentes $\mathrm{y} / \mathrm{o}$ del personal administrativo de las empresas industriales de la ciudad de Tacna manifiestan que de ninguna manera, la RSE es un asunto de "reputación social". En contraste, el $41,7 \%$ de los gerentes $y / o$ del personal 
administrativo, manifiestan que en alguna medida la RSE es considerada como un asunto de "reputación social", mientras que sólo el 33,3\% de los gerentes y/o del personal administrativo, dice que no está seguro, si la RSE sea un asunto de "reputación social".

Tabla $\mathbf{N}^{\circ}$ 05: Porcentaje de consideración de la RSE como un asunto de "reputación social".

\begin{tabular}{|c|r|r|r|}
\hline Categoría & Frecuencia & Porcentaje & $\begin{array}{r}\text { Porcentaje } \\
\text { acumulado }\end{array}$ \\
\hline En ninguna medida & 3 & 25,0 & 25,0 \\
\hline No estoy seguro (a) & 4 & 33,3 & 58,3 \\
\hline En alguna medida & 5 & 41,7 & 100,0 \\
\hline Total & 12 & 100,0 & \\
\hline
\end{tabular}

Fuente: Matriz de sistematización de datos.

\subsection{A qué nivel es percibida su organización como poseedora de reputación social positiva}

Como se observa en la tabla $\mathrm{N}^{\circ} 06$, el $50 \%$ de los gerentes $\mathrm{y} / \mathrm{o}$ del personal administrativo de las empresas industriales de la ciudad de Tacna consideran que en regular nivel es percibida su organización, como poseedora de reputación social positiva.

Asimismo, el 8,3\% de los gerentes y/o del personal administrativo de las empresas consideran que su organización es percibida a un bajo nivel, como poseedora de reputación social positiva. En contraste, el 25\% de los gerentes y/o del personal administrativo consideran que su organización es percibida, a un alto nivel, como poseedora de reputación social positiva, mientras que sólo el 16,7\% de los gerentes y/o del personal administrativo dice que su organización es percibida a un nivel moderado.

Tabla $\mathbf{N}^{\circ}$ 06: Porcentaje de nivel de percepción de la organización como poseedora de reputación social.

\begin{tabular}{|l|r|r|r|}
\hline \multicolumn{1}{|c|}{ Categoría } & Frecuencia & Porcentaje & $\begin{array}{r}\text { Porcentaje } \\
\text { acumulado }\end{array}$ \\
\hline A bajo nivel & 1 & 8,5 & 8,3 \\
\hline A regular nivel & 6 & 50,0 & 58,3 \\
\hline A un nivel moderado & 2 & 16,7 & 75,0 \\
\hline A un alto nivel & 3 & 25,0 & 100,0 \\
\hline Total & 12 & 100,0 & \\
\hline
\end{tabular}

Fuente: Matriz de sistematización de datos

\subsection{Inversión anual en acciones de RSE}

Como se observa en la tabla $\mathrm{N}^{\circ} 7$, el $58,3 \%$ de los gerentes y/o del personal administrativo de las empresas industriales de la ciudad de Tacna manifiestan que su organización invierte menos de $\$ 10$ mil anuales en acciones de RSE. En contraste, el $41,7 \%$ de los gerentes $\mathrm{y} / \mathrm{o}$ del personal administrativo dicen que su organización invierte entre $\$ 10$ y $\$ 20$ mil anuales en acciones de RSE.

Tabla $\mathbf{N}^{\circ}$ o7: Porcentaje de inversión anual en acciones de RSE.

\begin{tabular}{|l|r|r|r|}
\hline \multicolumn{1}{|c|}{ Categoría } & Frecuencia & Porcentaje & $\begin{array}{c}\text { Porcentaje } \\
\text { Acumulado }\end{array}$ \\
\hline Menos de $\$ 10 \mathrm{mil}$ & 7 & 58,3 & 58,3 \\
\hline $\begin{array}{l}\text { Entre } \$ 10 \text { y } \$ 20 \\
\text { mil }\end{array}$ & 5 & 41,7 & 100,0 \\
\hline Total & 12 & 100,0 & \\
\hline
\end{tabular}

Fuente: Matriz de sistematización de datos

3.8 ¿Mide la empresa el impacto de operaciones a nivel económico?

Como se observa en la tabla $\mathrm{N}^{\circ} 08$, el $41,7 \%$ de los gerentes y/o del personal administrativo de las empresas industriales de la ciudad de Tacna manifiestan que su organización mide el impacto de sus operaciones a nivel económico. En contraste, el $58,3 \%$ de los gerentes $\mathrm{y} / \mathrm{o}$ del personal administrativo manifiestan que su organización no mide el impacto de operaciones a nivel económico.

Tabla $\mathbf{N}^{\circ}$ o8: Porcentaje de impacto de operaciones de la empresa a nivel económico.

\begin{tabular}{|c|c|c|c|}
\hline Categoría & Frecuencia & $\begin{array}{c}\text { Porcentaj } \\
\text { e }\end{array}$ & $\begin{array}{c}\text { Porcentaje } \\
\text { acumulado }\end{array}$ \\
\hline $\mathrm{Si}$ & 5 & 41,7 & 41,7 \\
\hline No & 7 & 58,3 & 100,0 \\
\hline Total & 12 & 100,0 & \\
\hline
\end{tabular}

Fuente: Matriz de sistematización de datos.

\section{9. ¿Mide la empresa el impacto de sus operaciones a nivel ambiental?}

Tal como se observa en la tabla $\mathrm{N}^{\circ} 09$, el $66,7 \%$ de los gerentes y/o del personal administrativo de las empresas industriales de la ciudad de Tacna manifiestan que su organización mide el impacto de sus operaciones a nivel ambiental. En contraste, el $33,3 \%$ de los gerentes $y / o$ del personal administrativo manifiesta que su organización no mide el impacto de sus operaciones a nivel ambiental.

Tabla $\mathrm{N}^{\circ}$ 09: Porcentaje de impacto de operaciones de la empresa a nivel económico.

\begin{tabular}{|l|r|r|r|}
\hline Categoría & Frecuencia & Porcentaje & $\begin{array}{r}\text { Porcentaje } \\
\text { acumulado }\end{array}$ \\
\hline $\mathrm{Si}$ & 8 & 66,7 & 66,7 \\
\hline No & 4 & 33,3 & 100,0 \\
\hline Total & 12 & 100,0 & \\
\hline
\end{tabular}

Fuente: Matriz de sistematización de datos. 


\subsection{0. ¿Mide la empresa el impacto de sus operaciones a nivel social?}

Como se observa en la tabla $\mathrm{N}^{\circ} 10$, el $41,7 \%$ de los gerentes y/o del personal administrativo de las empresas industriales de la ciudad de Tacna manifiesta que su organización sí mide el impacto de sus operaciones a nivel social. En contraste, el $58,3 \%$ de los gerentes $\mathrm{y} / \mathrm{o}$ del personal administrativo manifiesta que su organización no mide el impacto de sus operaciones a nivel social.

Tabla $\mathrm{N}^{\mathbf{0}}$ 10: Porcentaje de impacto de operaciones de la empresa a nivel social.

\begin{tabular}{|l|r|r|r|}
\hline Categoría & Frecuencia & Porcentaje & $\begin{array}{r}\text { Porcentaje } \\
\text { acumulado }\end{array}$ \\
\hline $\mathrm{Si}$ & 5 & 41,7 & 41,7 \\
\hline No & 7 & 58,3 & 100,0 \\
\hline Total & 12 & 100,0 & \\
\hline
\end{tabular}

Fuente: Matriz de sistematización de datos.

\section{DISCUSIÓN}

Al emprender nuestro estudio nos propusimos evaluar la posible influencia de la RSE en los resultados económicos de las empresas industriales de la ciudad de Tacna.

$\mathrm{Al}$ respecto, es necesario precisar que el $58.3 \%$ de las empresas industriales de la ciudad de Tacna, sí tiene prácticas/políticas de RSE frente a un $41,7 \%$ de gerentes $y / o$ del personal administrativo de dichas empresas que no tiene prácticas/políticas de RSE. Por lo que es necesario referir que las empresas industriales de la ciudad de Tacna, en su mayoría, aplican prácticas o políticas de RSE.

El $25 \%$ de los encuestados consideran que están muy de acuerdo que la RSE es vital para la sustentabilidad y rentabilidad de cualquier empresa, y el 58,3\% de este grupo esta de acuerdo.

Asimismo, el $25 \%$ de los encuestados manifiestan que en ninguna medida consideran a la RSE, como un asunto de "reputación social". En contraste, el $41,7 \%$ de este grupo dice que en alguna medida la RSE es un asunto de "reputación social" y el $33,3 \%$ del conjunto, se manifiesta escéptico, pues está seguro si la RSE puede ser considerada como un asunto de "reputación social".

El $58,3 \%$ de las empresas encuestadas invierten menos de $\$ 10$ mil anuales en acciones de RSE. En contraste, el $41,7 \%$ de este grupo invierte entre $\$ 10$ y $\$ 20$ mil anuales en acciones de RSE.
Además, debemos destacar que, el $66,7 \%$ del conjunto de empresas industriales encuestadas manifiesta que ocasionalmente miden y evalúan sus programas y procesos de RSE; el 8,3\% nunca mide ni evalúa sus programas y procesos de RSE; mientras que el $25 \%$ de este grupo consideran que constantemente miden dicha práctica.

Por lo que es necesario referir que las empresas industriales de la ciudad Tacna, en su mayoría, aplican prácticas o políticas de RSE.

\section{CONCLUSIONES}

- Las empresas industriales de la ciudad Tacna, en su mayoría, aplican prácticas o políticas de RSE.

- Las empresas industriales de la ciudad de Tacna consideran a la RSE como un asunto de relaciones públicas muy importante, además, manifiestan que es vital para la sustentabilidad y rentabilidad de cualquier empresa. De otro lado, las referidas organizaciones de la ciudad de Tacna, consideran a la RSE como un asunto de "reputación social"; sin embargo, las mencionadas organizaciones precisan que su organización no presenta un alto nivel de reputación social positiva.

- En las empresas industriales de la ciudad de Tacna, las labores de RSE son asumidas de la siguiente forma: un área asume la responsabilidad total y dicha responsabilidad es compartida entre varias áreas de la organización.

- En las empresas industriales de la ciudad de Tacna, el nivel de inversión en RSE es bajo, el cual no alcanza a los $\$ 10$ mil dólares anuales, lo cual se traduce en la poca importancia económica que se le atribuye a dicha área.

- Las empresas industriales de la ciudad de Tacna miden y evalúan sus programas y procesos de Responsabilidad Social Empresarial. Asimismo, miden el impacto de sus operaciones a nivel económico, social y ambiental.

- Las empresas industriales de la ciudad de Tacna manifiestan que, después de aplicar las prácticas y/o políticas de Responsabilidad Empresarial, la organización ha mejorado sus resultados económicos. En general, las empresas declaran que los beneficios que esperaría para su organización mediante el trabajo en Responsabilidad Social Empresarial serían en el área social, ambiental e interna.

- Las empresas industriales de la ciudad de Tacna, en general, están dispuestas a realizar 
alianzas con otras empresas para implementar sus acciones de Responsabilidad Social Empresarial.

\section{REFERENCIAS BIBLIOGRÁFICAS}

\section{Asociación española de Contabilidad y Administración de Empresas (2004): Marco Conceptual de la Responsabilidad Social Corporativa, Documentos AECA, Serie Responsabilidad Social Corporativa, $\mathrm{N}^{\circ} \mathrm{O} 1$.}

Camacho, Ildefonso, et al. (2005). Etica de la empresa. Bilbao: Universidad Deusto. 319 p.

\section{Comisión de las Comunidades Europeas} (2001): Libro verde. Fomentar un marco europeo para la responsabilidad social de las empresas, Comisión Europea, Bruselas. URL: http://europa.eu.int/com/off/green/indexes.htm

Campos, Victoria, et al. (2003). Ética y Ciudadanía. Lecciones. Medellín: Ateneo Porfirio Barba Jacob. 158 p.

Carroll, Archie B. (1999). "Corporate Social Responsibility. Business and Society. pp 268285.

Centro Mexicano para la Filantropía (Cemefi, 2009), ¿Qué es el Cemefi? Información institucional, en URL: http://cemefi.org/ nosotros/informacion-institucional.html

Cortina, Adela, et al. (2003). Construir Confianza. Etica de la empresa en la sociedad de la información y las comunicaciones. Madrid: Editorial Trotta. 226 p.

Forética (2008). Informe Forética 2008. Evolución de la Responsabilidad Social de las empresas en España. URL: http://www.foretica.es/recursos/doc/Bibliote ca/Informes/36900_16121612200821230.pdf

Fundación Prohumana de Chile (2011). ¿Qué es la RSE? Responsabilidad Social de la Empresa, en URL: http://www.prohumana.cl/ index.php?option $=$ com_content\&task =view\&i $\mathrm{d}=4.4$ \&Intemid $=60$.

Garriga, Elizabet y MeleDomenech. (2004), Corporate Social Responsability theories: Mapping The Territory. Journal of Business Ethics. pp. 51-71.

Guidi, M. (2006) "Estado, Empresas, Sociedad Civil. Reflexiones a partir de una experiencia", Iberoforum, otoño, $\mathrm{N}^{\circ} \mathrm{II}$, año 1 .
ICONTEC. (2006). Tres anos construyendo un marco de normalización para la responsabilidad social en Colombia. Boletín Informativo. $30 \mathrm{p}$.

Klein, Naomi. (2005). No Logo: El poder de las marcas. Barcelona: Paidos. 559p.

Lozano, J.M. (2006): "De la responsabilidad social de la empresa (RSE) a la empresa responsable y sostenible", Papeles de economía española, pp.40-62.

Mercado P. y García P. (2007) "La responsabilidad social en empresas del valle de Toluca México. Un estudio exploratorio" Estudios gerenciales, vol.23, $\mathrm{N}^{\circ} 102$, eneromarzo.

Porter, Michael E. y Kramer Mark R. (2006). Estrategia y Sociedad: $\mathrm{El}$ vinculo entre ventaja competitiva y responsabilidad social corporativa, Harvard Business Review. pp.4257.

Restrepo F., Pablo y Trujillo M., Carolina(2007). La responsabilidad social empresarial como parte de la estrategia organizacional. Tesis de grado Maestría en Administración. Medellín: Universidad Eafit.

Server, R.J. y Villalonga, I. (2005): "La responsabilidad social Corporativa (RSC) y su gestión integrada", CIRIEC-España, Revista de Economía Pública, Social y Cooperativa, $\mathrm{N}^{\circ} 53$, pp. 137-161.

Vargas, J. (2006). Responsabilidad Social Empresarial (RSE) desde la perspectiva de los consumidores, Comisión Económica para América Latina y el Caribe (CEPAL), Naciones Unidas.

Correspondencia:

Luis Rocchetti Herrera

Ciudad Universitaria fundo "Los Granados"

Av. Miraflores s/n Tacna - Perú

Elizabeth Medina Soto

Ciudad Universitaria fundo "Los Granados" Av. Miraflores s/n Tacna - Perú

Jesús Olivera Cáceres

Ciudad Universitaria fundo "Los Granados" Av. Miraflores s/n Tacna-Perú

Gonzalo Gambini Quezada

Ciudad Universitaria fundo "Los Granados"

Av. Miraflores s/n Tacna - Perú 